\title{
Macrophage activation syndrome as a complication of rheumatologic disorders, a report from Iran
}

\section{SUMMARY}

The objective was to evaluate the clinical and laboratory manifestations and outcomes of the MAS cases in the context of systemic juvenile idiopathic arthritis (SJIA), systemic lupus erythematosus (SLE), Kawasaki disease, poly-articular juvenile idiopathic arthritis (PJIA).

Twenty consecutive patients diagnosed with MAS between 2005 and 2016 entered the study. The cases were divided into two groups: in the first, MAS emerged in the context of a previously diagnosed rheumatologic disease, while in the second, MAS was the first presentation of a rheumatologic disease. In the other classification, the cases were divided into recurrent and non-recurrent cases. Laboratory data were recorded at three times: before MAS attack, during MAS attack, and 1 month after discharge from hospital.

Nineteen cases with the median age of 5.9 (3.6-10) years entered the study. Four cases (21.1\%) showed recurrent attacks of MAS. MAS was the first presentation of disease in 10 cases. The median age of the patients in the underlying disease group (10 years) was significantly higher than in the first presentation group [4.5(1.7-6.1) years, $\mathrm{p}=0.003$ ]. The median fibrinogen value during MAS attack in the underlying disease group (601 $\mathrm{mg}$ / $\mathrm{dL})$ was also significantly higher than in the first presentation group $(174 \mathrm{mg} / \mathrm{dL}, \mathrm{p}=0.038)$. The platelet count during MAS attack in the recurrent group (30,500/microliter) was significantly lower than in the non-recurrent group $(135,000 /$ microliter, $\mathrm{p}=0.042)$.

Our series of MAS cases demonstrated an overview of the symptoms, signs, laboratory manifestations, treatment, and prognosis of these cases. The higher median fibrinogen in MAS in the underlying disease group revealed that a decreasing level of fibrinogen in chronic disease is more significant than a single cut off value. Indeed, the lower platelet count in the recurrent MAS group may indicate greater platelet consumption due to organomegaly. Early diagnosis and treatment may save the patients' lives.

Key words: Macrophage activation syndrome; systemic juvenile idiopathic arthritis; systemic lupus erythematosus; Kawasaki.

Reumatismo, 2019; 71 (4): 189-198

\section{INTRODUCTION}

$\mathrm{M}$ acrophage activation syndrome (MAS) is a severe and life-threatening complication in the context of autoimmune and auto-inflammatory disorders (1-3). The main disease in this category is systemic juvenile idiopathic arthritis (SJIA), but MAS may also appear in other diseases, such as Kawasaki disease (4), systemic lupus erythematosus (SLE) (5), and periodic fever syndromes $(2,3)$.
MAS may be induced by the flare-up of the underling disease or by a trigger factor such as infection, changes in the treatment, and treatment complications of biologic or other drugs (6-8). The patients may present with non-remitting fever, hepatosplenomegaly, lymphadenopathy, encephalopathy, coagulopathy, and even multi-organ failure in severe cases. The laboratory data may show pancytopenia, hypertriglyceridemia, hyperferritinemia, hypofibrinogenemia, and increase in transaminase levels (9). $\overline{\text { Corresponding author: }}$ Vahid Ziaee

Division of Pediatric Rheumatology, Children's Medical Center,

No. 62 Dr. Gharib St., Keshavarz Blvd, Tehran 14194, Iran E-mail: ziaee@tums.ac.ir 
Delayed diagnosis of MAS may lead to life-threatening complications and increase in mortality rate. Recent studies reported a mortality rate of $8 \%$ in this disorder $(10$, 11). Due to the importance of early diagnosis and treatment for MAS, many studies were performed to help earlier diagnosis of MAS and differentiate this disorder from other situations such as flare-up of underlying disease or infections (12-14).

This study evaluated the clinical and laboratory manifestations and outcomes of the MAS cases as an emergent complication in the context of SJIA, SLE, Kawasaki, polyarticular juvenile idiopathic arthritis (PJIA).

\section{MATERIALS AND METHODS}

Twenty consecutive patients diagnosed with MAS, requiring hospital admission in the Children Medical Center between 2005 and 2016, entered the prospective study. The patients over 18 years of age, patients that did not return for follow-up, and patients with diagnosis of primary hemophagocytic lymphohistiocytosis (HLH) were excluded from the study. One patient with resistance to treatment was excluded with the diagnosis of Griscelli syndrome according to the whole exome sequencing analysis. Because of the triggering effect of the infection for MAS in the cases with rheumatologic diseases, documented infection was not an exclusion criterion. The study was approved by Institutional Review Board of the Children Medical Center (Tehran University of Medical Sciences). The study and data collection were compliant with the principles of the Declaration of Helsinki. Informed consent was obtained from all patients.

The cases were selected according to the clinical and laboratory signs of MAS (9). Attacks of MAS can occur in the setting of SJIA, PJIA, SLE, and Kawasaki. In the admissions of flared up patients with underlying disease and patients with first manifestations unresponsive to treatment, the serial changes of complete blood count (CBC), ESR, CRP and laboratory data including ferritin, fibrinogen, triglycerides and liver function test were registered. All cases were required to meet both previous (9) and new criteria of Ravelli et al. (14) and Parodi et al. (4) All the MAS patients according to the previous criteria fulfilled the new Ravelli criteria (14). In Kawasaki disease the MAS patient fulfilled Ravelli's 2016 criteria $(14,15)$. For primary diagnosis of the background disease, the relevant criteria were used (16-18).

In history taking, the age and gender of the cases and the primary symptoms were recorded. Three time intervals were obtained from the patients' history and used in the analysis:

1) The time interval from the beginning of the underlying rheumatologic disease to the hospital admission. In patients where MAS was the first presentation of rheumatologic disease, this time was considered zero.

2) The time interval from deteriorating general condition to hospital admission.

3) The time interval from hospital admission to definite diagnosis and starting treatment.

Patients' drug histories were also obtained. The patients in the MAS group were divided according to their drug history into four groups:

1) Active on drugs.

2) Active without drugs.

3) Remission on drugs.

4) Remission off drugs.

In the physical examination, abnormal signs of the patients were noted. The tympanic body temperature was measured in all patients. Abdominal sonography and echocardiography were performed for all patients.

Laboratory data, including white blood cells (WBC), polymorphonuclear (PMN), lymphocyte, hemoglobin, platelet count, erythrocyte sedimentation rate (ESR), and C-reactive protein (CRP), were recorded at three times: before MAS attack (previous records were searched at the initial diagnosis of background disease), during MAS attack, and 1 month after discharge from hospital. In the cases where MAS was the first presentation of disease, the previous records and consequently, the first group of the laboratory data were not available. Ferritin, fibrinogen, alanine aminotransferase 
(ALT), aspartate aminotransferase (AST), alkaline phosphatase, lactate dehydrogenase (LDH), serum sodium, prothrombin time (PT), partial thromboplastin time (PTT), and international normalized ratio (INR) were recorded only during the MAS attack. Changes in the laboratory data (for example WBC1, WBC2, WBC3) were also evaluated.

The eligible patients were followed until at least 12 months. The outcome of the diseases was classified into complete resolution, resistance to treatment, recurrence, and death. If within three months after complete resolution of the first attack, the laboratory manifestations of MAS recurred, the disease was classified in the recurrence category.

At admission, all the patients were treated according to the underlying disease. The MAS patients were treated with methylprednisolone pulses for three days. The resistant and recurrent MAS patients were treated according to HLH 2004 guidelines with dexamethasone, cyclosporine and etoposide (19).

Statistical analysis was done by SPSS version 22 (SPSS Inc. Chicago, IL). Categorical variables were shown as a number (percentage). Continuous variables were shown as median (interquartile range). Two classifications were used in the analysis. In one of the classifications (as noted before), the cases were divided in two groups:

1) Where MAS occurred in the setting of previously diagnosed rheumatologic disease (underlying disease group).

2) Where MAS was the first presentation of rheumatologic disease (first presentation group).

In the other classification, the cases were divided into recurrent and non-recurrent cases. All symptoms, signs, laboratory data, and outcome results were compared between the above groups. A chi-squared test was used to compare the categorical variables between two groups. The MannWhitney U test was used to compare continuous variables between 2 groups. $p<0.05$ was considered significant.

\section{RESULTS}

Nineteen cases [11 (57.2\%) males] with the median age of 5.9 (3.6-10) years entered the study. Demographic and baseline characteristics of the patients are shown in Table I.

Table I - The characteristic data of patients. The quantitative data (data and durations) are shown as median [interquartile range (IQR)]. The qualitative data are shown as a number (percentage).

\begin{tabular}{|c|c|c|}
\hline \multicolumn{2}{|l|}{ Parameter } & \multirow{2}{*}{$\begin{array}{c}\text { Frequency/Mean } \\
11(57.9 \%) \\
8(42.1 \%)\end{array}$} \\
\hline Gender & $\begin{array}{l}\text { Male } \\
\text { Female }\end{array}$ & \\
\hline Age (years) & & $5.9(3.6-10)$ \\
\hline Recurrent attacks & $\begin{array}{l}\text { Non-recurrent MAS } \\
\text { Recurrent MAS }\end{array}$ & $\begin{array}{c}15(78.9 \%) \\
4(21.1 \%)\end{array}$ \\
\hline Diagnosis & $\begin{array}{l}\text { SJIA } \\
\text { Polyarticular JIA } \\
\text { Kawasaki } \\
\text { SLE }\end{array}$ & $\begin{array}{c}9(47.3 \%) \\
4(21 \%) \\
5(26.3 \%) \\
1(5.2 \%)\end{array}$ \\
\hline Time of attack (MAS) & $\begin{array}{l}\text { First presentation } \\
\text { On underlying disease }\end{array}$ & $\begin{array}{c}10(52.6 \%) \\
9(47.4 \%)\end{array}$ \\
\hline Disease situation & $\begin{array}{l}\text { Active on drugs } \\
\text { Active without drugs } \\
\text { Remission on drug }\end{array}$ & $\begin{array}{c}8(42.1 \%) \\
10(52.6 \%) \\
1(5.2 \%)\end{array}$ \\
\hline Duration of basic disease (months) & & $24(4-60)$ \\
\hline Duration of acute symptoms (days) & & $21(12-60)$ \\
\hline Duration between admission to treatment (days) & & $2(2-7.2)$ \\
\hline
\end{tabular}

MAS: Macrophage Activation Syndrome, SD: standard deviation, SJIA: systemic onset juvenile idiopathic arthritis, PJIA: polyarticular juvenile idiopathic arthritis, and SLE: systemic lupus erythematosus. 
Four cases $(21.1 \%)$ showed recurrent attacks of MAS. The second attack occurred at the median interval of 9.5 (5-28) months from first attack. Of the recurrent cases, 3 cases occurred in the setting of SJIA. Of these 3 cases, 2 died (one due to noncompliance with treatment and one due to late referral). In the fourth case, the MAS occurred in the setting of Kawasaki disease. Four attacks of MAS were diagnosed in this case. In one of these 4 attacks, Guillain-Barre syndrome occurred concurrently. Immunologic evaluations were therefore performed and a diagnosis of Bruton agammaglobulinemia was made.

As shown in Table II, MAS was the first presentation of disease in 10 cases. Of these cases, 3 developed SJIA, 5 developed Kawasaki (including the Bruton case), and one developed SLE. These 10 cases had not received any treatment before diagnosis of MAS. The other 9 cases were under

Table II - The signs, sonography and echocardiography data of macrophage activation syndrome patients.

\begin{tabular}{|l|c|c|}
\hline Symptom & Positive & Negative \\
\hline Fever & $16(84.2 \%)$ & $3(15.7 \%)$ \\
\hline Hepatosplenomegaly & $7(36.8 \%)$ & \\
Hepatomegaly & $4(21 \%)$ & $7(36.8 \%)$ \\
Splenomegaly & $1(5.2 \%)$ & \\
\hline Lymphadenopathy & $3(15.7 \%)$ & $16(84.2 \%)$ \\
\hline Rash & $10(52.6 \%)$ & $9(47.4 \%)$ \\
\hline Hemorrhagic disorders & $4(21.5 \%)$ & $15(78.9 \%)$ \\
\hline Loss of consciousness & $5(26.3 \%)$ & $14(73.68 \%)$ \\
\hline Serositis & & \\
Pleural effusion & $8(42.1 \%)$ & \\
Pericardial effusion & $6(31.5 \%)$ & $4(21 \%)$ \\
Ascites & $1(5.2 \%)$ & \\
Total & $15(78.9 \%)$ & \\
\hline Respiratory distress & $8(42.1 \%)$ & $11(57.8 \%)$ \\
\hline Active arthritis & $5(26.3 \%)$ & $14(73.6 \%)$ \\
\hline Arthralgia & $3(15.7 \%)$ & $17(89.4 \%)$ \\
\hline Cardiac involvement & & \\
Pericardial effusion & $6(31.57 \%)$ & \\
Coronary dilation & $1(5.2 \%)$ & \\
Coronary aneurysm & $1(5.2 \%)$ & $7(36.84 \%)$ \\
Coronary dilation and LV dysfunction & $1(5.2 \%)$ & \\
PE and LV dysfunction & $1(5.2 \%)$ & \\
PE and LV dysfunction and coronary dilation & $1(5.2 \%)$ & \\
PE and LV dysfunction and PH & $1(5.2 \%)$ & \\
Total & $12(63.15 \%)$ & \\
\hline
\end{tabular}

PE: pericardial effusion; LV: left ventricle; $\mathrm{PH}$ : pulmonary hypertension. treatment for the underlying disease when they developed MAS. In one case, the underlying disease (SJIA) was under control and the drugs were discontinued. Due to hepatitis A infection, MAS developed in this case. In this study, all cases of Kawasaki were in the first presentation group.

The clinical manifestations of the patients are shown in Table II. Sixteen cases had fever. The fever in these patients was persistent and high-grade. Three cases did not have fever. The MAS developed in 2 cases in the setting of PJIA. As demonstrated in this table, 10 cases had cardiac involvement. Of these 12 cases, 4 cases with left ventricular (LV) dysfunction required intensive care unit admission. Three of these 4 cases died due to LV dysfunction.

The laboratory data of the patients in 3 stages, before MAS attack, during MAS attack, and after MAS attack, are shown in Table III. The comparison of these data was illustrated in another article completely (13). The treatment of MAS cases is shown in Table IV. As shown in this table, all the cases received corticosteroid treatment. Cyclosporine was in the treatment regimen of 13 cases. Two cases showed resistance to treatment. These cases had persistent fever, respiratory and neurologic manifestations, and LV dysfunction. According to the 2004 HLH treatment protocol, these cases underwent treatment with dexamethasone, cyclosporine, and etoposide. For one of these 2 cases, a preliminary diagnosis of HLH was made. According to whole exon sequencing test, the diagnosis of Griscelli syndrome was confirmed.

Sixteen patients underwent bone marrow aspiration. In 5 cases, hemophagocytic macrophages were found in the aspiration. Six patients showed hypercellular marrow. The other cases had normal bone marrow aspiration.

The outcome of the patients included: 4 recurrent cases, 2 resistant to treatment, and 3 deaths (including 2 recurrent cases). Thirteen cases showed complete improvement after treatment.

The median age of the patients in the underlying disease group was significantly higher than in the first presentation group 
Table III - The median [interquartile range (IQR)] of laboratory data before, during and after MAS attack.

\begin{tabular}{|c|c|c|c|}
\hline Parameter & Before MAS & MAS Attack & After MAS \\
\hline WBC $\left(\times 10^{3} / \mathrm{uL}\right)$ & $13,900(8,300-23,100)$ & $4,250(2,380-11,360)$ & $12,240(7,385-21,805)$ \\
\hline PMN (x103/uL) & $8,789(4,853-15,436)$ & $3,119(1,667-13,727)$ & $7,300(4,616-19,100)$ \\
\hline LYMPH (x103/uL) & $3,394(1,891-4,549)$ & $1,344(1,181-1,820)$ & $2,219(1,222-5,907)$ \\
\hline $\mathrm{Hgb}(\mathrm{g} / \mathrm{dL})$ & $8.8(8.3-10.5)$ & $7.7(6.8-8.8)$ & $8.8(7.8-9.9)$ \\
\hline PLT (/microliter) & $225,500(157,500-429,250)$ & $121,000(46,000-139,000)$ & $326,000(218,000-380,000)$ \\
\hline $\operatorname{ESR}(\mathrm{mm} / \mathrm{h})$ & $56(40-100)$ & $25.5(14-42)$ & $22(17-59)$ \\
\hline $\mathrm{CRP}(\mathrm{mg} / \mathrm{L})$ & $32(16-59)$ & $120(60-214)$ & $20(6-40)$ \\
\hline ALT (u/L) & - & $107(45-204)$ & - \\
\hline AST (u/L) & - & $117(41-498)$ & - \\
\hline $\operatorname{ALP}(u / L)$ & - & $316(242-650)$ & - \\
\hline ALB (gr/dL) & - & $2.8(2.5-3.3)$ & - \\
\hline Total protein (gr/dL) & - & $5.9(4.7-7.2)$ & - \\
\hline Bil total $(\mathrm{mg} / \mathrm{dL})$ & - & $2.9(0.6-7.3)$ & - \\
\hline Bil direct $(\mathrm{mg} / \mathrm{dL})$ & - & $1.4(0.2-5.9)$ & - \\
\hline Ferritin $(\mathrm{ng} / \mathrm{mL})$ & - & $18,678(8,000-113,000)$ & - \\
\hline Fibrinogen (mg/dL) & & $227(160-402)$ & - \\
\hline $\mathrm{LDH}(\mathrm{lu} / \mathrm{L})$ & - & $2,037(714-3,568)$ & - \\
\hline $\mathrm{BUN}(\mathrm{mg} / \mathrm{dL})$ & - & $10.5(9-28.7)$ & - \\
\hline $\mathrm{Cr}(\mathrm{mg} / \mathrm{dL})$ & - & $0.5(0.4-0.7)$ & - \\
\hline $\mathrm{Na}$ (meq/L) & - & $132(128-134)$ & - \\
\hline $\mathrm{TG}(\mathrm{mg} / \mathrm{dL})$ & - & 312 (199-438) & - \\
\hline Chol (mg/dL) & - & 174 (147-301) & - \\
\hline PT & $-\infty$ & $16.7(13.8-20.3)$ & - \\
\hline $\mathrm{PTT}$ & - & $40(36-51)$ & - \\
\hline INR & - & $1.69(1.2-2.32)$ & - \\
\hline
\end{tabular}

MMAS: Macrophage Activation Syndrome, WBC: white blood cells, PMN: polymorphonuclear, Lymph: lymphocytes, Hgb: hemoglobin, PLT: platelet, ESR: erythrocyte sedimentation rate, CRP: C-reactive protein, ALT: alanine aminotransferase, AST: aspartate aminotransferase, ALP: alkaline phosphatase, ALB: albumin, Total Pr: total protein, Bil T: total bilirubin, Bil D: direct bilirubin, LDH: lactate dehydrogenase, BUN: blood urea nitrogen, Cr: creatinine, Na: natrium, TG: triglycerides, Chol: cholesterol, PT: prothrombin time, PTT: partial prothrombin time, and INR: international normalized ratio.

The previous laboratory records: WBC1, PMN1, and so forth.

The laboratory data at the onset of MAS (MAS group): WBC2, PMN2, and so forth.

The laboratory data 1 month after discharge from the hospital: WBC3, PMN3, and so forth.

Table IV - Treatment of MAS cases in this study.

\begin{tabular}{|l|c|}
\hline Treatment & N. \\
\hline Methylprednisolone pulse & 5 \\
\hline Methylprednisolone pulse + cyclosporine & 5 \\
\hline $\begin{array}{l}\text { Methylprednisolone pulse + cyclosporine } \\
+ \text { IVIG }\end{array}$ & 4 \\
\hline $\begin{array}{l}\text { Methylprednisolone + IVIG + cyclosporine } \\
+ \text { Infliximab }\end{array}$ & 1 \\
\hline IVIG + Cyclophosphamide + prednisolone & 3 \\
\hline HLH protocol 2004 & 1 \\
\hline Total & 19 \\
\hline
\end{tabular}

(10 range: 6.4-13.1 vs 4.5 range: 1.7-6.1 years, $\mathrm{p}=0.003)$. The median fibrinogen value during MAS attack in the underlying disease group (601, range: 227-1289 $\mathrm{mg} / \mathrm{dL}$ ) was also significantly higher than in the first presentation group (174, range: $130-315 \mathrm{mg} / \mathrm{dL})(\mathrm{p}=0.038)$. Other symptoms, signs, laboratory data, and outcome results were not significantly different between these 2 groups.

The platelet count during MAS attacks in the recurrent group $(30,500$ range: $12,750-$ 
$65,500) /$ microliter) was significantly lower than in the non-recurrent group $(135,000$, range: $\quad(64,000-153,000) /$ microliter $)$ $(\mathrm{p}=0.042)$.

Other symptoms, signs, laboratory data, and outcome results were not significantly different between these 2 groups.

\section{DISCUSSION AND CONCLUSIONS}

The present study evaluated the clinical and laboratory manifestations and outcomes of the MAS cases in the setting of SJIA, SLE, Kawasaki, PJIA. To our knowledge, in the other studies, the MAS in SJIA, SLE, and Kawasaki disease (15) were evaluated (11, $14,20)$. In this study, the clinical manifestations, the serial changes of laboratory data, treatment, outcomes, the recurrent MAS patients and the MAS presentations as the first manifestations are recorded.

The male/female ratio in our study (57.9/42.1) was the reverse of that observed in other studies (21). This finding might be due to inclusion of MAS cases other than SJIA. In other SJIA studies, males and females showed equal proportions (22) and Kawasaki disease showed higher prevalence in males (23).

In our study, the recurrent cases were evaluated in a separate group. To our knowledge, in other studies, the recurrent cases were not evaluated separately.

Recurrent MAS could be due to gene variants in the cytolytic pathway $(24,25)$. Canna and colleagues reported NLRC4 gene in a case with recurrent attacks of MAS (26). The recurrent cases of MAS may be a separate autoinflammatory disorder that shows periodic manifestations (27) or a genetic disorder in HLH could be linked to MAS in the cytolysis process (28).

The evaluation of this concept requires further studies.

In our study, the median mean time between the beginning of the underlying disease and the beginning of the MAS attack was 24 months. This interval was much lower (3.5 months) in the Minoia et al. study (11). The cause of this finding might be the longer follow-up - about two and a half years - of the underlying disease. More cases of MAS with longer time intervals might be diagnosed, therefore. Furthermore, in our study, in $60 \%$ of the cases, MAS was the first presentation of the underlying disease. This percentage was $22 \%$ in the Minoia et al. study (11). In these cases, this interval was considered as zero. The main cause of this finding was that acute Kawasaki disease cases represented about $25 \%$ of our MAS patients.

The main feature of the fever in MAS is its persistent, not spiky, nature. In our study, 2 cases did not have fever. Both cases had had PJIA for several years and received anti-TNF drugs. These drugs might be the cause for the absence of fever in these cases. One of the cytokines responsible for inducing fever is TNF- $\alpha$ (29). Suppression of this cytokine might reduce the fever. In addition, anti-TNF drugs were proposed in some studies as triggering factors in MAS $(30,31)$. The evaluation of this concept requires further studies.

In our study, cardiac involvement occurred more than in other studies of MAS cases (Table II) $(11,32)$. Similarly to other studies, in the cases with cardiac involvement, pericardial effusion was the most common finding.

The second common finding in these cases was LV dysfunction, probably due to inclusion of Kawasaki cases. In the previous studies, infiltration of the phagocytes in the myocardium was proposed as a cause of LV dysfunction in MAS (33). In addition, the cause of death in all 3 cases in our study was LV dysfunction. No renal involvement was found in our cases. This finding is in contrast with other studies of MAS that showed the worst prognosis in the cases with renal involvement (34). On the other hand, the systemic scores proved to have significant role in the prognostic factors, while renal involvements were not included (35). Better evaluation of the prognostic factors requires further studies. In this study, all the cases received corticosteroid treatment. Corticosteroids are the first line of treatment in MAS cases $(20,36)$. Some of the cases improved with corticosteroid alone. In the cases with se- 
vere symptoms and signs of decreased consciousness, cyclosporine was added to the steroid at the beginning. Cyclosporine is the most important treatment after steroids for MAS $(36,38)$. If response to the treatment was inadequate, the treatment was continued according to the $2004 \mathrm{HLH}$ protocol (19). In the MAS cases associated with infection or Kawasaki disease, IVIG, with its immunoregulatory features, is an appropriate treatment $(39,40)$. In the resistant MAS in SLE cases, cyclophosphamide can be used in combination with steroid (41).

In bone marrow aspiration, as in 5 cases in our study, observation of hemophagocytes can confirm the diagnosis in suspicious cases. In some of the MAS cases, as in 6 cases in our study, the bone marrow may be hypercellular, without any hemophagocyte. In the bone marrow, hypercellularity with normal megakaryocytes demonstrates that the cytopenia is not due to inadequate production, but is due to increased cellular destruction by phagocytes in the inflammatory locations (42).

The median age of the patients in the underlying disease group was significantly higher than in the first presentation group. As noted, all cases of Kawasaki were in the first presentation group. At presentation, the Kawasaki cases generally are younger than those with with other diseases $(22,43$, 44). This could explain the lower median age in the first presentation group.

The laboratory findings of the MAS cases were fully discussed in our previous article (13). The laboratory findings in this study were in accordance with our previous study (13) and that of Ravelli et al. (14). The median fibrinogen value during an MAS attack in the underlying disease group was significantly higher than in the first presentation group. Fibrinogen value is a marker of inflammation. These higher values might be due to more severe and chronic inflammation and resultant higher fibrinogen in the underlying disease group (45). In the flare up of the underlying disease, when the fibrinogen level decreases from high level to normal level, MAS should therefore be considered (10-13).
In addition, the platelet count during MAS attack in the recurrent group was significantly lower than in the non-recurrent group. As noted, recurrent MAS may be an auto-inflammatory disorder. In auto-inflammatory disorders, thrombocytopenia may occur due to the role of platelets in innate immunity (46). On the other hand, hypercellularity and normal megakaryocytes in the bone marrow of MAS patients, determine consumption of leukocytes, erythrocytes, and platelets in the inflammatory site as well as phagocytic destruction $(47,48)$. Organomegaly was detected in all patients with recurrent MAS. This can explain the lower median platelet count in this group.

The limitations of our study were low sample size, absence of cellular and molecular (sIL2R $\alpha$, s CD163 and genetic evaluation) diagnosis for MAS, and poor availability of biologic drugs for MAS treatment. In the years that our study was progressing, further research on the immunopathogenesis of MAS took place and new advances occurred in MAS treatment $(49,50)$. These may lead to new therapeutic targets and improve the outcomes (51). Due to poor availability of biologic drugs in our country, these drugs were less widely used in the treatment of underlying rheumatologic diseases and MAS.

In conclusion, in rheumatologic disorder cases with pancytopenia, hyperferritinemia, and increased serum transaminases, MAS should be considered. The higher median fibrinogen in MAS in the underlying disease group revealed that a decreasing level of fibrinogen in chronic disease is more significant than a single cut off value. Indeed, the lower platelet count in the recurrent MAS group may reveal greater platelet consumption due to organomegaly in this group. Early diagnosis and treatment may save the patients' lives.

\section{List of abbreviations}

MAS: Macrophage activation syndrome SJIA: systemic juvenile idiopathic arthritis SLE: systemic lupus erythematosus

PJIA: poly-articular juvenile idiopathic arthritis 
HLH: hemophagocytic lymphohistiocytosis WBC: white blood cells PMN: polymorphonuclear ESR: erythrocyte sedimentation rate CRP: C-reactive protein ALT: alanine aminotransferase AST: aspartate aminotransferase LDH: lactate dehydrogenase PT: prothrombin time PTT: partial thromboplastin time INR: international normalized ratio LV: left ventricular

\section{Ethical approval}

All authors state that the research reported in the paper was undertaken in compliance with the Helsinki Declaration and the International Principles governing research on human beings. This study was approved by the local hospital ethical committee (in Children's Medical Center, Pediatrics Center of Excellence, Tehran, Iran).

\section{Availability of data and materials}

The data used to support the findings of this study are available from the corresponding author upon request.

\section{Funding. Nil.}

\section{Contributions}

RA, participated in the acquisition of data, manuscript preparation and critical revision of the manuscript; PS, participated in the concept and design of the study and interpretation of findings; $\mathrm{AM}$, participated in manuscript preparation and interpretation of findings; FE, participated in the acquisition of data; VZ, concept and design of the study, data collection, analysis and interpretation and critical revision of the manuscript All authors read and approved the final manuscript.

\section{Acknowledgements}

The authors would like to thank Dr. M. Morradinejad and F. Tahghighi for her contribution in the data gathering of this study.

\section{Competing interests}

All authors have no conflict or competing of interest to declare.

\section{REFERENCES}

1. Avcin T, Tse SM, Schneider R, et al. Macrophage activation syndrome as the presenting manifestation of rheumatic diseases in childhood. J Pediatr. 2006; 148: 683-6.

2. Rigante D, Capoluongo E, Bertoni B, et al. First report of macrophage activation syndrome in hyperimmunoglobulinemia $\mathrm{D}$ with periodic fever syndrome. Arthritis Rheum. 2007; 56: 658-61.

3. Rossi-Semerano L, Hermeziu B, Fabre M, Kone-Paut I. Macrophage activation syndrome revealing familial Mediterranean fever. Arthritis Care Res (Hoboken). 2011; 63: 780-3.

4. Simonini G, Pagnini I, Innocenti L, et al. Macrophage activation syndrome/hemophagocytic lymphohistiocytosis and Kawasaki disease [letter]. Pediatr Blood Cancer. 2010; 55: 592.

5. Parodi A, Davi S, Pringe AB, et al, for the Lupus Working Group of the Paediatric Rheumatology European Society. Macrophage activation syndrome in juvenile systemic lupus erythematosus: a multinational multicenter study of thirty-eight patients. Arthritis Rheum. 2009; 60: 3388-99.

6. Grom AA, Mellins ED. Macrophage activation syndrome: advances towards understanding pathogenesis. Curr Opin Rheumatol. 2010; 22: 561-6.

7. Buoncompagni A, Loy A, Sala I, Ravelli A. The paradox of macrophage activation syndrome triggered by biologic medications. Pediatr Rhematol Online J. 2005; 3: 70-3.

8. Lurati A, Teruzzi B, Salmaso A, et al. Macrophage activation syndrome (MAS) during anti-IL1 therapy (anakinra) in a patient affected by systemic juvenile arthritis (soJIA): a report and review of the literature. Pediatr Rheumatol Online J. 2005; 3: 79-85.

9. Ravelli A, Magni-Manzoni S, Pistorio A, et al. Preliminary diagnostic guidelines for macrophage activation syndrome complicating systemic juvenile idio-pathic arthritis. J Pediatr. 2005; 146: 598-604.

10. Minoia F, Davi S, Horne A, et al.; on behalf of the Pediatric Rheumatology International Trials Organization, the Childhood Arthritis and Rheumatology Research Alliance, the Pediatric Rheumatology Collaborative Study Group, and the Histiocyte Society. Clinical features, treatment, and outcome of macrophage activation syndrome complicating systemic juvenile idiopathic arthritis: a multinational, multicenter study of 362 patients. Arthritis Rheumatol. 2014; 66: 3160-9.

11. Minoia F, Davi S, Horne A, et al, Pediatric Rheumatology International Trials Organization, Childhood Arthritis and Rheumatology Research Alliance, Pediatric Rheumatology Collaborative Study Group, Histiocyte So- 
ciety. Dissecting the heterogeneity of macrophage activation syndrome complicating systemic juvenile idiopathic arthritis. J Rheumatol. 2015; 42: 994-1001.

12. Davì S, Consolaro A, Guseinova D, et al. An international consensus survey of diagnostic criteria for macrophage activation syndrome in systemic juvenile idiopathic arthritis. J Rheumatol. 2011; 38: 764-8.

13. Assari R, Ziaee V, Mirmohammadsadeghi A, Moradinejad MH. Dynamic changes, cut-off points, sensitivity, and specificity of laboratory data to differentiate macrophage activation syndrome from active disease. Dis Mark. 2015; 2015: 424381.

14. Ravelli A, Minoia F, Davì S, et al. 2016 Classification Criteria for Macrophage Activation Syndrome Complicating Systemic Juvenile Idiopathic Arthritis: A European League Against Rheumatism/American College of Rheumatology/Paediatric Rheumatology International Trials Organisation Collaborative Initiative. Ann Rheum Dis. 2016; 75: 481-9.

15. Garcia-Pavon S, Yamazaki-Nakashimada MA, Baez M, et al. Kawasaki disease complicated with macrophage activation syndrome: A systematic review. J Pediatr Hematol Oncol. 2017; 39: 445-51.

16. Petty RE, Southwood TR, Manners P, et al. International League of Associations for Rheumatology classification of juvenile idiopathic arthritis: second revision, Edmonton, 2001. J Rheumatology. 2004; 31: 390-2.

17. Newburger JW, Takahashi M, Gerber MA, et al. Diagnosis, treatment, and long-term management of Kawasaki disease: a statement for health professionals from the Committee on Rheumatic Fever, Endocarditis and Kawasaki Disease, Council on Cardiovascular Disease in the Young, American Heart Association. Circulation. 2004; 110: 2747-71.

18. Hochberg MC. Updating the American College of Rheumatology revised criteria for the classification of systemic lupus erythematosus. Arthrit Rheum. 1997; 40: 1725.

19. Henter JI, Horne A, Aricó M, et al. HLH-2004: diagnostic and therapeutic guidelines for hemophagocytic lymphohistiocytosis, Pediatr Blood Cancer. 2007; 48: 124-31.

20. Boom V, Anton J, Lahdenne P, et al. Evidencebased diagnosis and treatment of macrophage activation syndrome in systemic juvenile idiopathic arthritis. Pediatr Rheumatol J. 2015; 13: 55.

21. Lehmberg K, Pink I, Eulenburg C, et al. Differentiating macrophage activation syndrome in systemic juvenile idiopathic arthritis from other forms of hemophagocytic lymphohistiocytosis. J Pediatr. 2013; 162: 1245-51.

22. Behrens EM, Beukelman T, Gallo L, et al. Evaluation of the presentation of systemic on- set juvenile rheumatoid arthritis: data from the Pennsylvania Systemic Onset Juvenile Arthritis Registry (PASOJAR). J Rheumatol. 2008; 35: 343-8.

23. Ha S, Seo GH, Kim KY, Kim DS. Epidemiologic Study on Kawasaki Disease in Korea, 2007-2014: Based on Health Insurance Review; Assessment Service Claims. J Korean. 2016; 31:1445-9.

24. Kaufman KM, Linghu B, Szustakowski JD, et al. Whole Exome Sequencing Reveals Overlap Between Macrophage Activation Syndrome in Systemic Juvenile Idiopathic Arthritis and Familial Hemophagocytic Lymphohistiocytosis. Arthritis Rheumatol. 2014; 66: 3486-95.

25. Zhang M, Behrens EM, Atkinson TP, et al. Genetic Defects in Cytolysis in Macrophage Activation Syndrome. Curr Rheumatol Rep. 2014; 16: 439.

26. Canna SW, de Jesus AA, Gouni S, et al. An activating NLRC4 inflammasome mutation causes autoinflammation with recurrent macrophage activation syndrome. Nat Genet. 2014; 46: 1140-6.

27. Rigante D, Emmi G, Fastiggi M, et al. Macrophage activation syndrome in the course of monogenic autoinflammatory disorders. Clin Rheumatol. 2015; 34: 1333-9.

28. Vastert SJ, Wijk RV, D’Urbano LE, et al. Mutations in the perforin gene can be linked to macrophage activation syndrome in patients with systemic onset juvenile idiopathic arthritis. Rheumatology. 2010; 49: 441-9.

29. Sundgren-Andersson AK, Ostlund P, Bartfai T. IL-6 is essential in TNF-alpha-induced fever. Am J Physiol. 1998; 275: 2028-34.

30. Ramanan AV, Schneider R. Macrophage activation syndrome following initiation of etanercept in a child with systemic onset juvenile rheumatoid arthritis. J Rheumatol. 2003; 30: 401-3.

31. Sandhu C, Chesney A, Piliotis E, et al. Macrophage activation syndrome after etanercept treatment. J Rheumatol. 2007; 34: 241-2.

32. Stabile A, Bertoni B, Ansuini V, et al. The clinical spectrum and treatment options of macrophage activation syndrome in the pediatric age. Eur Rev Med Pharmacol Sci. 2006; 10: 53-9.

33. Stephan JL, Zeller J, Hubert P, et al. Macrophage activation syndrome and rheumatic disease in childhood: a report of four new cases. Clin Exp Rheumatol. 1993; 11: 451-6.

34. Sawhney S, Woo P, Murray KJ. Macrophage activation syndrome: a potentially fatal complication of rheumatic disorders. Arch Dis Child. 2001; 85: 421-6.

35. Ruscitti P, Rago C, Breda L, et al. Macrophage activation syndrome in Still's disease: analysis of clinical characteristics and survival in paediatric and adult patients. Clin Rheumatol. 2017; 36: 2839-45. 
36. Lin CI, Yu HH, Lee JH, et al. Clinical analysis of macrophage activation syndrome in pediatric patients with autoimmune diseases. Clin Rheumatol. 2012; 31: 1223-30.

37. Stephan JL, Kone-Paut I, Galambrun C, et al. Reactive haemophagocytic syndrome in children with inflammatory disorders. A retrospective study of 24 patients. Rheumatology (Oxford). 2001; 40: 1285-92.

38. Kounami S, Yoshiyama M, Nakayama K, et al. Macrophage activation syndrome in children with systemic-onset juvenile chronic arthritis. Acta Haematol. 2005; 113: 124-9.

39. Larroche C, Bruneel F, Andre MH, et al. Intravenously administered gamma-globulins in reactive hemophagocytic syndrome. Ann Med Interne. 2000; 151: 533-9.

40. Emmenegger U, Frey U, Reimers A, et al. Hyperferritinemia as indicator for intravenous immunoglobulin treatment in reactive macrophage activation syndromes. Am J Hematol. 2001; 68: 4-10.

41. Torres Jiménez A, Solís Vallejo E, Zeferino Cruz M. Macrophage activation syndrome as the initial manifestation of severe juvenile onset systemic lupus erythematosus. Favorable response to cyclophosphamide. Reumatol Clin. 2014; 10: 331-5.

42. Grom AA. Natural killer cell dysfunction: A common pathway in systemic-onset juvenile arthritis, macrophage activation syndrome, and hemophagocytic lymphohistiocytosis? Arthritis Rheum. 2004; 50: 689-98.

43. Ogilvie EM, Fife MS, Thompson SD, et al. The $-174 \mathrm{G}$ allele of the interleukin- 6 gene confers susceptibility to systemic arthritis in children: a multicenter study using simplex and multiplexjuvenile idiopathic arthritis families. Arthritis Rheum. 2003; 48: 3202-6.

44. Saurenmann RK, Rose JB, Tyrrell, et al. Epi- demiology of juvenile idiopathic arthritis in a multicenter cohort: ethnicity as a risk factor. Arthritis Rheum. 2007; 56: 1974-84.

45. Ravelli A. Expert consensus on dynamics of laboratory tests for diagnosis of macrophage activation syndrome complicating systemic juvenile idiopathic arthritis. RMD Open 2016; 2: e000161.

46. Klinger MH, Jelkmann W. Role of blood platelets in infection and inflammation. J Interferon Cytokine Res. 2002; 22: 913-22.

47. Stephan JL, Kone-Paut I, Galambrun C, et al. Reactive Haemophagocytic syndrome in children with inflammatory disorders. A retrospective study of 24 patients. Rheumatology (Oxford). 2001; 40: 1285-92.

48. Grom AA, NK dysfunction: a common pathway in systemic onset juvenile rheumatoid arthritis, macrophage activation syndrome, and hemophagocytic lymphohistiocytosis. Arthritis Rheum. 2004; 50; 689-98.

49. Miettunen PM, Narendran A, Jayanthan A, et al. Successful treatment of severe paediatric rheumatic disease-associated macrophage activation syndrome with interleukin-1 inhibition following conventional immunosuppressive therapy: case series with 12 patients. Rheumatology (Oxford). 2011; 50: 417-9.

50. ClinicalTrials.gov. Ong-term Follow-up of HLH Patients Who Received Treatment With NI-050, an Anti-interferon Gamma Monoclonal Antibody. NLM Identifier: NCT02069899. Available from: http://clinicaltrials.gov/show/ NCT02069899 Accessed: 12 Apr 2015.

51. Ruscitti P, Cipriani P, Benedetto PD, et al. Advances in immunopathogenesis of macrophage activation syndrome during rheumatic inflammatory diseases: toward new therapeutic targets? Expert Rev Clin Immunol. 2017; 13: 1041-7. 